\title{
Probabilistic-based Approach for Fast Impairments-aware RWA in All-Optical Networks
}

\author{
L. Velasco ${ }^{1 *}$, A. Jirattigalachote ${ }^{2}$, P. Monti ${ }^{2}$, L. Wosinska ${ }^{2}$, S. Spadaro ${ }^{1}$, G. Junyent ${ }^{1}$ \\ ${ }^{1}$ Universitat Politècnica de Catalunya (UPC), Jordi Girona 1-3, 08034 Barcelona, Spain \\ ${ }^{2}$ The Royal Institute of Technology (KTH), Electrum 229, Isafjordsgatan 22, 16440 Kista, Sweden \\ *Tel: (34) 9340169 99, Fax: (34) 9340170 55, e-mail:lvelasco@ac.upc.edu
}

\begin{abstract}
We devised a probabilistic model to compute the number of used wavelengths in every link of a transparent optical network. Based on this model, a novel approach for impairments-aware RWA algorithm is proposed and evaluated.

(C) 2009 Optical Society of America

OCIS codes: (060.0060) Fiber optics and optical communications; (060.4250) Networks
\end{abstract}

\section{Introduction}

Physical layer impairments can be classified into linear and nonlinear effects. Linear impairments are independent of the signal power and affect each of the wavelengths individually, whereas nonlinear impairments affect not only each optical channel individually but they also cause disturbance and interference between them. The most important linear impairments are: fiber attenuation, Amplifier Spontaneous Emission (ASE) noise, Chromatic Dispersion (CD) (or Group Velocity Dispersion (GVD)), and Polarization Mode Dispersion (PMD). The most important non-linear impairments are: Self Phase Modulation (SPM), Cross Phase Modulation (XPM), and Four Wave Mixing (FWM).

The incorporation of physical layer impairments information in the routing and wavelength assignment (RWA) problem for transparent optical networks has recently received a lot of attention, leading to the development of a number of impairment-aware RWA (Ia-RWA) algorithms [1-4]. The general idea behind these algorithms is to compute the Q-factor of the candidate lightpaths and accept only those with a value above a given threshold. However, in a distributed approach, when nonlinear impairments are considered, an Ia-RWA algorithm needs to know the current state of the network to compute the Q-factor of a given lightpath. This translates into the need to have some extensions to the Generalized Multi-Protocol Label Switching (GMPLS) protocol set [5] in order to provide the necessary information for a distributed and accurate $I a-R W A$ computation.

A different approach is to consider impairments as fixed penalties for each link assuming a fully loaded system, referred to as the worst case scenario [4]. Advantages of this approach are: (i) very short lightpath setup time (the Q-factor can be pre-computed beforehand for each link), and (ii) no need for GMPLS protocol extensions. However, the blocking probability obtained using this approach might be unnecessarily high.

In order to prevent the prohibitively high control overhead of the (accurate) distributed approach while providing an improved network efficiency compared with the fixed penalties approach this paper presents a probabilistic model to compute the distribution of the number of used wavelength in every link in the network as a function of the offered load. Then, using the proposed model, we calculate the probability of having more than $\lambda_{l}$ wavelengths for a given load $l$. Moreover, we define a threshold for this probability (i.e. we define the highest allowable probability of having more than $\lambda_{l}$ wavelengths and refer to it as the accuracy threshold) and we refer to $\lambda_{l}$ as the probabilistic maximum number of wavelengths. Finally, we compute the Q-factor of a lightpath assuming that $\lambda_{l}$ wavelengths are being used in every link. We call this strategy the impairments-aware probabilistic (worst) case (Ia-PC). Ia-PC does not require any GMPLS protocol extension, which can significantly reduce network control complexity. In addition a minimal lightpaths set-up delay can be ensured since the Q-factor can be pre-computed. Simulation results show that the $I a-P C$ resulting blocking probability is comparable with the one obtained by already existing I $a-R W A$ which considers the actual network state.

\section{Probabilistic Model}

Let $G(N, E, W)$ be the graph of an optical network, where $N, E$ and $W$ represent the set of nodes, links and wavelengths per link, respectively. Let us define $H$ as the average hop length of a lightpath. Without loss of generality, we consider that every lightpath is routed using the shortest path. Besides, we assume the traffic to be uniformly distributed in the network. Then, the average length of the shortest paths in a mesh network can be approximated by $H=((|N|-2) /(\delta-1))^{1 / 2}$, where $\delta$ is the average nodal degree [6]. The average number of used wavelengths in a link can then be computed as $\lambda=C \cdot H /|E|$, where $C$ represents the average number of established lightpaths for a given offered load.

The work reported in this paper was supported by the Network of Excellence "Building the Future Optical Network in Europe" (BONE), funded by the European Commission through the 7th ICT-Framework Programme. Moreover, it was supported by the Spanish science ministry through the project ENGINE (TEC200802634). 
As already mentioned in the Sec. 1, we are interested in modeling the cumulative distribution $P\left(X \leq \lambda_{l}\right)$ for the maximum number of used wavelengths in every link of the graph. At first, we tried the random variable $X$ following a binomial distribution, $X \sim B(|W|, \alpha)$, where $\alpha=\lambda / W \mid$ is the average ratio of used wavelengths. After a number of simulations we concluded that the binomial distribution adjusts fine for low loads but not for medium and high loads. For this reason we propose an alternative model for the complementary cumulative distribution:

$$
P\left(Y>\lambda_{l}\right)=\alpha \cdot\left(\frac{1}{2} \cdot \frac{|W|-\lambda_{l}}{|W|-\lambda}\right)+(1-\alpha) \cdot\left[1-P\left(X \leq \lambda_{l}\right)\right]
$$

The model is a sum of two terms: the first term is a line containing two points: $P(Y>|W|)=0$ and $P(Y>\lambda)=1 / 2$, whereas the second term is modeled by the binomial distribution previously defined. Both terms are weighted by the parameter $\alpha$, and then for low loads we obtain a distribution close to the binomial while for medium and high loads we obtain a mixture of both terms.

The model has been validated over different networks with different average nodal degrees and in all cases it provides good results for accuracy threshold lower than $50 \%$ for a wide range of offered loads. We have fixed the required accuracy threshold to $10 \%$, and then for every offered load we obtain the values of $\lambda_{l}$. As an example, we obtained the values represented in Table 1 for the 16-Node European Optical Network (EON) with 16 wavelengths per link.

Table 1. Max number of used Wavelengths (accuracy threshold $\leq 10 \%$ ) against the offered load

\begin{tabular}{lllllllllllll}
\hline Offered Load (Erlangs) & 8 & 16 & 24 & 32 & 40 & 48 & 56 & 64 & 72 & 80 & 88 & 96 \\
Max used wavelengths $\left(\lambda_{l}\right)$ & 3 & 5 & 7 & 9 & 10 & 11 & 12 & 13 & 14 & 14 & 15 & 16 \\
\hline
\end{tabular}

\section{Impairments model}

In our study, the effect of both linear and nonlinear physical layer impairments is quantified by using the quality factor Q [7]. The considered Q-factor includes PMD, ASE noise, the combined SPM/GVD and optical filtering effects, XPM, and FWM. ASE, FWM and XPM are calculated assuming that they follow a Gaussian distribution. For the combined SPM/GVD and optical filtering effects, they are quantified through an eye closure metric calculated on the most degraded bit-pattern. Furthermore, the power penalty due to PMD is calculated basing on the length of lightpath, bit rate and lightpath PMD parameter. The Q-factor of a lightpath is given according to the equation (2),

$$
Q=\frac{p e n_{\text {eye }} \cdot P_{\text {transmitter }}}{p e n_{P M D} \cdot \sqrt{\sigma_{A S E}^{2}+\sigma_{X P M}^{2}+\sigma_{F W M}^{2}}}
$$

where $P_{\text {transmitter }}$, pen eye $_{\text {e }}$ pen ${ }_{P M D}, \sigma_{A S E}^{2}, \sigma_{X P M}^{2}, \sigma_{F W M}^{2}$, are the power of transmitted signal, the relative eye closure attributed to SPM/GVD and optical filtering effects, the power penalty due to PMD, the electrical variance of ASE noise, and the electrical variance of XPM and FWM induced degradation, respectively.

\section{Performance Evaluation and Conclusion}

We have analysed how the considered impairments affect the final Q-factor value for a lightpath. SPM/GVD and PMD produce an eye closure penalty that is a function of the length of the lightpath, while variance produced by ASE, XPM, and FWM depends on the channel spacing (we assume channel spacing of 50GHz). We have quantified the total variance experienced by a lightpath when another lightpath uses a wavelength on a shared optical link. The total variance is very low when the two wavelengths on the shared link are separated by several unused channels (i.e. when the channel spacing between the two selected wavelengths is large). On the other hand the variance has a sharp increase when the number of free wavelengths in between decreases (i.e. when the selected wavelengths are close to each other), and it becomes very high when the two lightpaths are using contiguous wavelengths. Therefore, the search order used for the wavelength assignment algorithm should be taken into account. In our experiments, we use the search order represented in Table 2, which spreads the used wavelengths as much as possible. The chosen order minimizes the total variance and increases the lightpath Q-factor value. Fig. 1 shows the contribution to total variance of a lightpath using the $8^{\text {th }}$ wavelength over a link of $540 \mathrm{~km}$, when the number of used wavelengths is increased, and using the assignment order given in Table 2. As shown, the maximum slope of the graph corresponds to contiguous wavelengths assignment.

Table 2. Wavelengths Search Order

\begin{tabular}{lllllllllllllllll}
\hline Wavelength \# & 1 & 2 & 3 & 4 & 5 & 6 & 7 & 8 & 9 & 10 & 11 & 12 & 13 & 14 & 15 & 16 \\
Search order & 6 & 11 & 13 & 4 & 15 & 2 & 9 & 1 & 10 & 3 & 16 & 5 & 14 & 7 & 12 & 8 \\
\hline
\end{tabular}

We have conducted simulations over the 16-Node European Optical Network (EON) to compare the performance of three different impairment-aware strategies. The first strategy is the one which computes lightpaths Q-factor using the actual state of the network, referred to as the Impairments-aware Current-State (Ia-CS). 


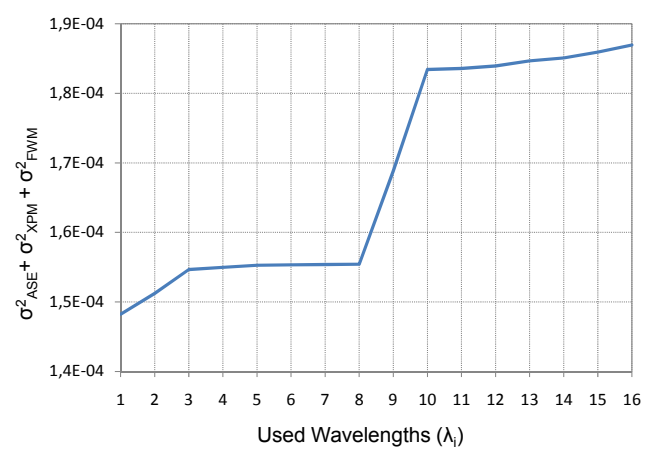

Fig. 1. ASE, XPM and FWM variance for a link as a function of the number of used wavelengths.

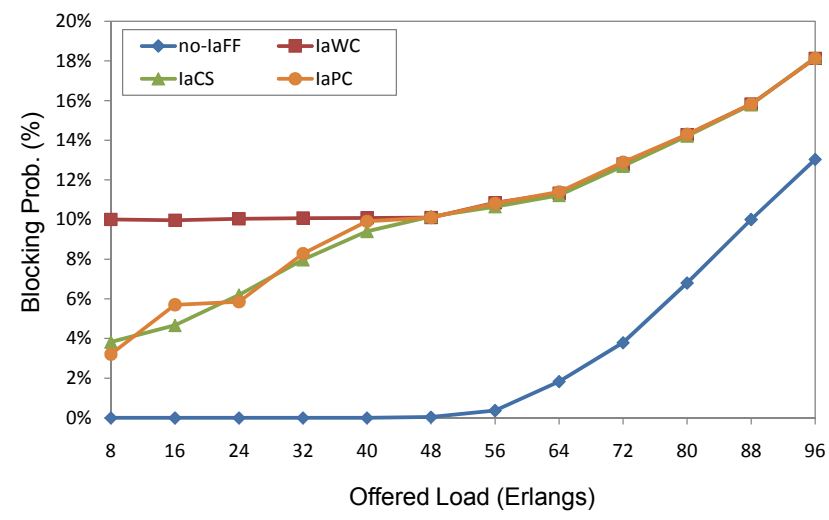

Fig. 2. Blocking probability against the offered load to the network.

This strategy needs the complete information regarding resource usage in order to compute the Q-factor of a new lightpath. If the Q-factor is better than a given threshold (we use $Q_{-}$threshold $=7.4$ which is equivalent to a BER $\approx 6.810^{-14}$ ), the lightpath is temporary routed. Then the Q-factor of each lightpaths already established, sharing one or more links with the new one, is recomputed and reevaluated. If all Q-factors are above the threshold, the new lightpath is established, otherwise it is blocked.

The second strategy is the one considering the worst case scenario, referred to as the Impairments-aware Worst-Case $(I a-W C)$ which assumes that all wavelengths are in use. Note that this strategy neither needs to know the actual state of the network to compute lightpaths Q-factor nor Q-factor reevaluation is necessary.

Finally, the proposed probabilistic approach $(I a-P C)$, where the expected maximum number of used wavelengths is obtained using the probabilistic model presented in Sec. 2.

Fig. 2 shows the obtained overall blocking probability (lack of resources plus impairments under the given threshold) as a function of the offered load, for the three previously described strategies. Each simulation was ended when the $95 \%$ confidence interval was within $5 \%$ of the point estimate. In the figure, blocking probability obtained with no-Ia-RWA, which uses the first-fit (FF) heuristic for wavelength assignment, is also shown for comparison. Results from no-Ia-FF represent the lower bound of blocking probability since this strategy does not block lightpath requests because of impairments.

As shown, $I a-W C$ provides the worst performance in terms of blocking probability, while $I a-C S$ provides the best one. The performance of both approaches becomes very similar under high offered loads. Ia-PC provides results very close to $I a-C S$, where its jerk-like behavior is a consequence of the fixed $\lambda_{l}$ used for every offered load.

It is worth noting that both approaches, $I a-C S$ and $I a-W C$, ensure that every established lightpath will experience a BER better than or equal to the threshold. However, that is not the case for the Ia-PC approach where this constraint is relaxed. To quantify that relaxation we use a merit figure closely related with the concept of availability. In a similar way, a lightpath becomes unavailable when it experiences a BER higher than the threshold, otherwise it remains available. Therefore, let us define the Impairments-aware availability $(I a-A)$ as the ratio between the time that a lightpath is available and the total time the lightpath is established in the network.

The drawback deriving from using the probabilistic approach is quantified in terms of Ia-A (or its complementary unavailability, $I a-U$ ). Table 3 provides the obtained unavailability results. As shown, $I a-U$ values are better than $10^{-3}$ in all cases, being negligible for medium and high loads.

Table 3. Impairments unavailability against the offered load

\begin{tabular}{lcccccccccccc}
\hline Offered Load (Erlangs) & 8 & 16 & 24 & 32 & 40 & 48 & 56 & 64 & 72 & 80 & 88 & 96 \\
Unavailability $(\mathbf{I a}-\boldsymbol{U})$ & $3.7 \mathrm{E}-4$ & $9.9 \mathrm{E}-6$ & $6.5 \mathrm{E}-4$ & 0 & 0 & 0 & 0 & 0 & 0 & 0 & 0 & 0 \\
\hline
\end{tabular}

Therefore, assuming this really small penalty for the network performance, we can conclude that it is possible to include impairments awareness in RWA computation without paying the complexity penalty and requiring standardize extensions to the GMPLS protocols set, as needed by the Ia-CS approach.

\section{References}

1. R. Cardillo, et al., "Considering Transmission Impairments in Wavelength Routed Networks", ONDM 2005.

2. E. Salvadori, et al. IEEE/OSA J. Lightw. Technol., 27 (2009).

3. S. Azodolmolky, et al., Computer Networks 53 (2009).

4. S. Pachnicke, et al., OSA J. Opt. Netw. 7 (2008).

5. E. Mannie, "Generalized Multi-Protocol Label Switching (GMPLS) Architecture", RFC-3945 (2004).

6. S.K. Korotky, IEEE/OSA J. Lightw. Technol., 22 (2004).

7. A. Jirattigalachote, et al., "Impairment Aware Routing with Service Differentiation in Heterogeneous WDM Networks", to appear in ACP 2009. 